\title{
Virtual Circular Geofences for Points and Regions of Interests with Spatial Context
}

\author{
Hoshito Tokita ${ }^{\mathrm{a}}$, Masatoshi Arikawa ${ }^{\mathrm{a}, *}$, Ruochen $\mathrm{Si}^{\mathrm{b}}$, Ren Sato ${ }^{\mathrm{a}}$, Ryodai Tayama ${ }^{\mathrm{c}}$, \\ and Akinori Takahashi ${ }^{\mathrm{a}}$ \\ ${ }^{a}$ Graduate School of Engineering Science, Akita University,m8019514@s.akita-u.ac.jp, arikawa@ie.akita-u.ac.jp, \\ m8019511@s.akita-u.ac.jp,akinori@ie.akita-u.ac.jp \\ ${ }^{b}$ Center for Spatial Information Science, the University of Tokyo, si@csis.tokyo-u.ac.jp \\ ${ }^{c}$ I. M. Service Co., Ltd., r tayama@ims-net.org
}

Keywords: Geofences, Design and Analysis, Smartphones, Mobile Mapping, Push Services, LBS

\begin{abstract}
:
GPS is useful for realizing push services on mobile devices including smartphones against users while their moving outside. The basic mechanism of location-based push services (LBS's) is generally based on a concept of geofence (Fig. 1). A geofence is defined by a spatial region. When a user would come into a geofence, a location-based service would generate an event of "Enter" on a mobile device with GPS. On the other hand, when a user would go out from a geofence, an event of "Exit" would be generated on it. The most popular and simplest shape of a geofence is a circular. In the case of a circular geofence, the condition of determine if a user is inside or outside is simple and only calculating a distance between a user's position and a central point of a circular. It is important to keep high efficiency in generating events of multiple geofences up to changes of a user's location and overlaps of geofences.
\end{abstract}

A conventional circular geofence of a point of interest (POI) generally shares the same point of a POI as a central point of it (Fig. 1(A)). The conventional circular geofences are useful and often used in many applications, but it may cover inappropriate places where users cannot appreciate the POI because of walls, buildings, trees, stairs, hills, rivers and so on. In order to avoid the inappropriate push services, a radius of the geofence is often adjusted smaller. The adjusted smaller radius could decrease problems of activations of geofences in appropriate locations, but other problems of inactivation of geofences where users are in right places to appreciate LBS, could happen. There are almost no perfect solutions of setting a radius of a circular geofence to satisfy all situation. The design of balancing a radius of each geofence is important to maximize users' satisfaction or to minimize users' unpleasantness for location-based push services. The weak points of the conventional circular geofence must come from the constraint of the same positon between a POI's position and a central positon of the circular region of a geofence. We proposed a new concept of virtual circular geofence, the central position of which need not be the same as a POI's position (Fig. 2). A good example of setting a virtual circular geofence is a viewpoint of a POI (Fig. 2(D')). When there are multiple viewpoints for a POI, multiple virtual geofences could be created for them. The advantage of virtual circular geofences is to minimize activation in inappropriate locations as well as inactivation in appropriate locations because regions of geofences can be easily and naturally set in right positions with right radiuses. On the other hand, the disadvantage of virtual circular geofences is that the system of geofence becomes a bit complex, but not so because of only a simple extension of geofence.

Virtual circular geofences can be applied to applications of regions of interests such as parks, campuses, and shopping malls (Fig. 1(B) (C)). When the conventional circular geofence is applied to regions of interests, it might be difficult to set the positon and the radius of the circular for arbitrary regions. As the results, inappropriate activation or inactivation of geofences could happen because of unfitting regions between them. Multiple virtual circular geofences could comprise a better region corresponding to a region of interests (Fig. 2 (C')). Another example of using VCGF for gateways of a region of interest. Two VCGF's are needed to comprise a gateway of ROI (Fig. 2 (E')). One is an outer region of a gateway, and the other is an inner region of it. If a user would come into a ROI, firstly the outer VCGF would activate, and then the inner VCGF would be activate. A sequential events from "outer" to "inner" would generates "check-in" of a ROI. On the other hand, "inner" to "outer" would make "check-out" of a ROI. The gateway use of VCGF is useful, but they could happens an accident of keeping "check-in" and "check-out" if a user could not be detected by a gateway VCGF's. In case, a conventional CGF is useful for exception handling of the accident.

There are following types of storytelling for walking tours.

(1) storytelling for a point

(2) storytelling for a narrow region

(3) storytelling for a wide region

(4) storytelling of a list structure for a wide region

(5) storytelling of a hierarchical structure for a wide region 
VCGF can be used to design of push service of audio tours to fit the above structure of storytelling. If different VCGF's overlaps, VCGF of a narrow region should be treated as a more prior information than one of a wide region. If a user appreciate a push service from a certain POI, the corresponding VCGF's might be removed because providing the same information is useless for users. We are experimenting to induce good places and good radiuses for POI's according to the trajectory data of tourists' movement as Fig. 3. We executed two tests; one is to record users' movement log data using a mobile map application with only pull services by users' operations and the other one with push services based on geofences including VCGF (Fig. 4).

As the conclusion, VCGF could be revealed by the experiments to be more useful, natural and practical than conventional CGF for real word applications. We have been developing more practical prototype systems and are planning to prove the feasibility of our proposal using more scientific data.

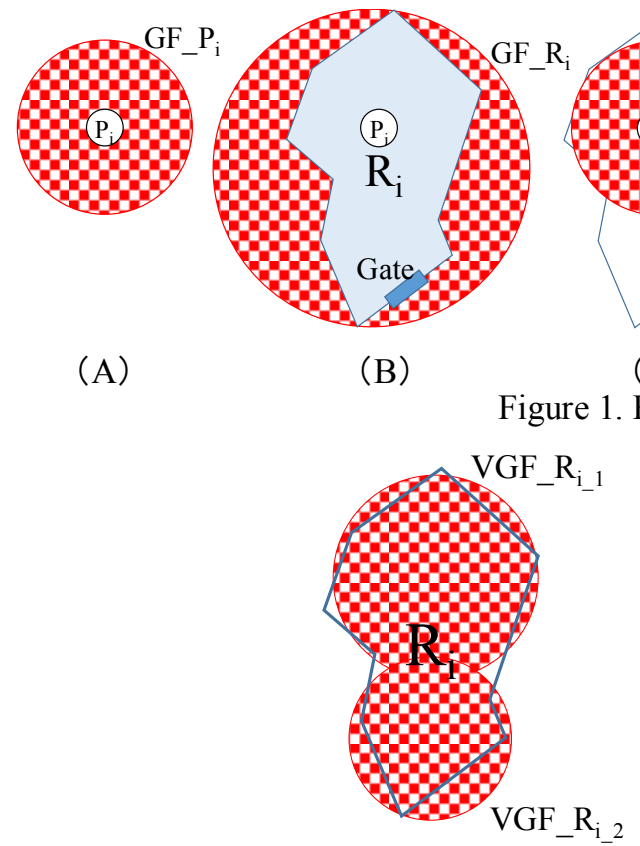

(C')

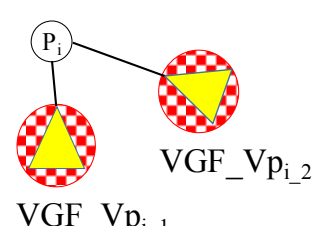

(D')

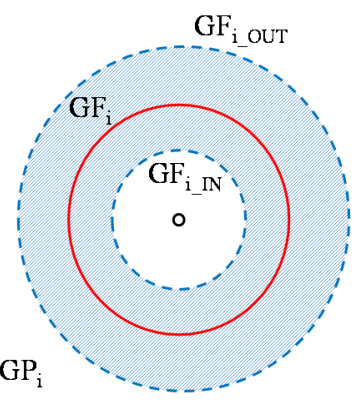

(F)

(C)

(D)

(E)

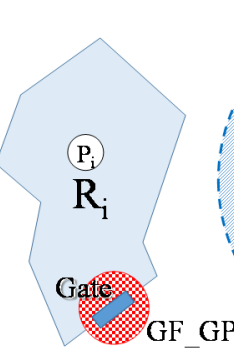

(E's)

Figure 2. Examples of Virtual Circular Geofences (VCGF's)

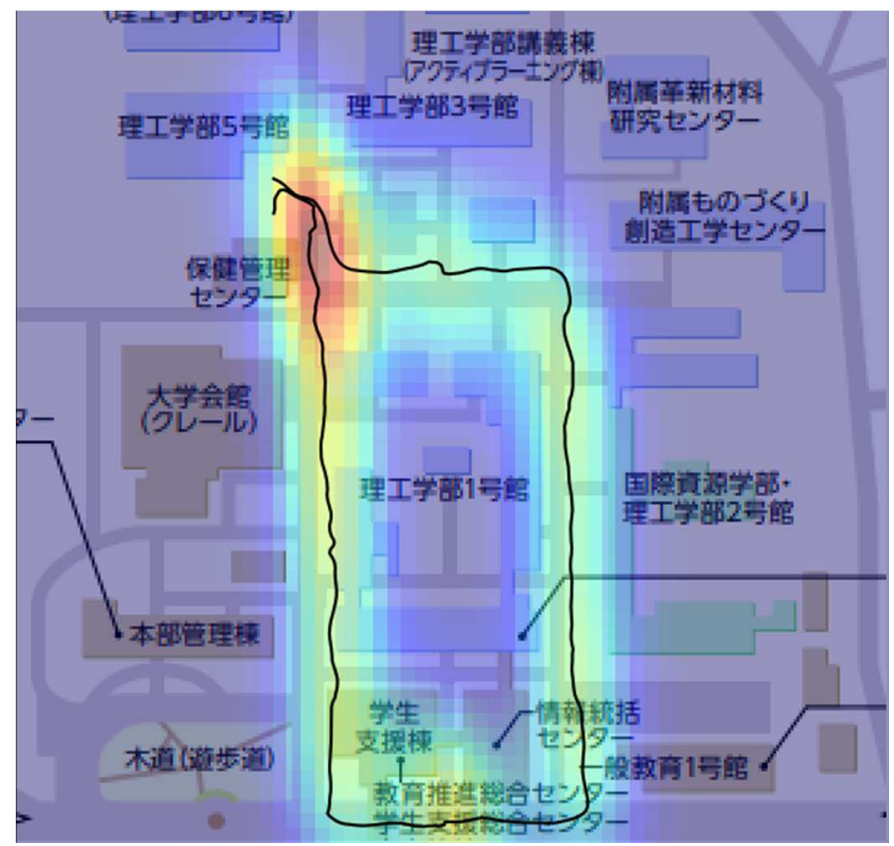

Figure 3. Visualization of a GPS Trajectory of Representing a User's Behavior

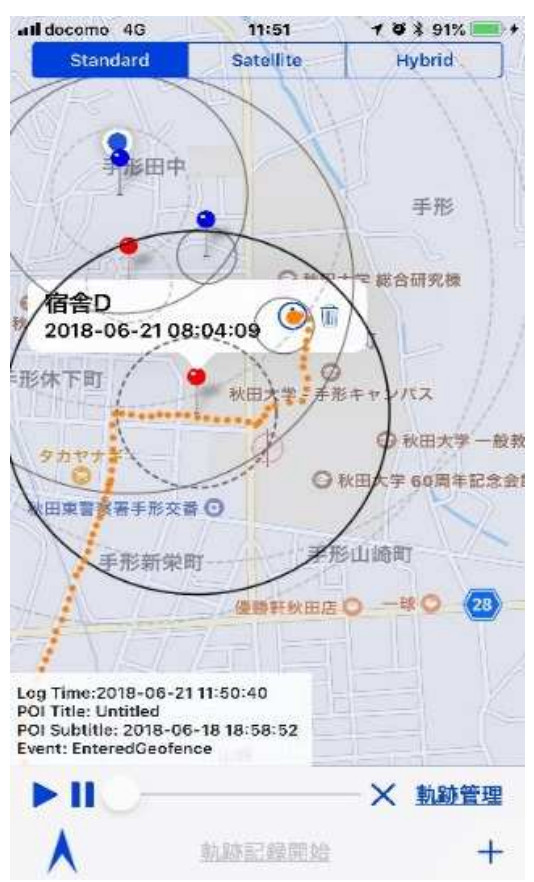

Figure 4. Prototype of an Experiment System for Testing of Positions and Radiuses of Circular Geofences 